\title{
NCCN Guidelines Updates: Prostate Cancer and Prostate Cancer Early Detection
}

\author{
Presented by Peter H. Carroll, MD, MPH, and James L. Mohler, MD
}

\section{Abstract}

Peter H. Carroll, MD, MPH, and James L. Mohler, MD, updated attendees on what is new in the 2018 NCCN Guidelines for Prostate Cancer Early Detection and for Prostate Cancer, respectively. Their presentations touched on new screening recommendations, shared decisionmaking, risk stratification, the role of genomic and molecular testing, active surveillance, and newer systemic treatments.

J Natl Compr Canc Netw 2018;16(5.5):620-623 doi: 10.6004/jnccn.2018.0036

"Th here may be a cancer subject that is more controversial than the screening and early detection of prostate cancer, but I don't know what that is," said Peter H. Carroll, MD, MPH, Professor and Chair, Department of Urology, UCSF Helen Diller Family Comprehensive Cancer Center, speaking at the NCCN 23rd Annual Conference. Dr. Carroll, Chair of the NCCN Guidelines for Prostate Cancer Early Detection, reviewed updates to the 2018 guidelines.

Screening/early detection guidelines for prostate cancer from NCCN, American Urological Association (AUA), American Cancer Society (ACS), and the US Preventive Services Task Force (USPSTF) differ in several ways, but these guidelines are coming closer to agreement, and in 2018, they all support shared decision-making between patients and clinicians.

The 2018 NCCN Guidelines for Prostate Cancer Early Detection continue to support early detection in well-informed healthy men and to recommend beginning baseline screening at 45 years of age, which is younger than the recommendations set forth in the

Presented by Peter H. Carroll, MD, MPH, Department of Urology, UCSF Helen Diller Family Comprehensive Cancer Center, San Francisco, California, and James L. Mohler, MD, Department of Urology, Roswell Park Comprehensive Cancer Center, Buffalo, New York.

Drs. Carroll and Mohler have disclosed that they have no financial interests, arrangements, affiliations, or commercial interests with the manufacturers of any products discussed in this article or their competitors.

Correspondence: Peter H. Carroll, MD, MPH, Department of Urology, UCSF Helen Diller Family Comprehensive Cancer Center, 1432 Lake Street, San Francisco, CA 94118. Email: peter.carroll@ucsf.edu; and James L. Mohler, MD, Department of Urology, Roswell Park Comprehensive Cancer Center, Elm and Carlson Streets, Buffalo, NY 14263. Email: james.mohler@roswellpark.org other 3 sets of guidelines. These updated NCCN Guidelines also support germline testing, consistent with other NCCN Guidelines.

"The NCCN Guidelines acknowledge that the optimal screening of high-risk patients is not completely known," Dr. Carroll stated.

A key feature of the updated guidelines is that they provide alternatives to routine biopsy in men with elevated prostate-specific antigen (PSA) levels, such as serum biomarker testing and parametric MRI. Further, they support the use of active surveillance in men identified to have low-risk cancers, linked to and compliant with the NCCN Guidelines for Prostate Cancer.

Dr. Carroll acknowledged that in the 1990s and 2000s, routine screening was poorly implemented, with downsides that included overscreening of older men, underscreening of younger men, overtreatment of lowrisk disease, and undertreatment of high-risk disease. "Despite driving mortality down by $>50 \%$, the cost of routine screening was too much in entirely avoidable treatment and its attendant side effects," he stated. "We need to screen smarter. The issue is not whether PSA testing and screening save lives," he stated.

NCCN, AUA, ACS, and USPSTF recommend different target ages for screening, populations to test, and thresholds for biopsy. NCCN recommends PSA screening for all men aged 45 to 75 years and discourages screening in patients aged $>75$ years; biopsy is recommended when the PSA level is $>3.0 \mathrm{ng} / \mathrm{mL}$. The USP STF has changed its controversial recommendation against routine PSA screening for all men. The new recommendation proposes individualized screening decisions based on consultation with a clinician, consider- 
Prostate Cancer and Prostate Cancer Early Detection

ing risks versus benefits and personal preferences (ie, shared decision-making). NCCN, AUA, and ACS guidelines also incorporate shared decision-making.

\section{Shared Decision-Making}

Risk factors that should be incorporated into shared decision-making include age, ethnicity (African Americans are known to be at higher risk for aggressive prostate cancer), family history, and a high risk for germline mutations, especially BRCA1/2.

Principles involved in shared decision-making include understanding that the goal of early detection is to identify aggressive prostate cancer early and cure it before it spreads outside the prostate, and communicating to patients that most prostate cancers identified by screening are indolent and can be safely managed using active surveillance. ${ }^{1}$

"The Achilles' heel of screening is overdetection and overtreatment of indolent tumors," Dr. Carroll emphasized. "The value of establishing an early baseline is to identify patients who don't need to be screened every 2 years," he stated. "If the PSA is $<1.0 \mathrm{ng} / \mathrm{mL}$ at age 60 years, the likelihood of prostate cancer death is $<0.3 \%$. Ninety percent of prostate cancer deaths occur in men in the top 10\% for PSA." A low baseline PSA value suggests that less intense testing is sufficient, whereas a high level is a strong predictor of lethal disease, he explained.

"Don't interpret PSA in a vacuum," he continued. Other factors to be considered include age, ethnicity, family history, digital rectal examination results, prior prostate biopsy findings, and whether the patient is taking finasteride.

\section{Alternatives to Biopsy}

Alternatives to immediate biopsy can improve the specificity of PSA testing, especially in men who want to avoid biopsy (Figure 1). The 2018 NCCN Guidelines recommend that clinicians consider the use of biomarkers and multiparametric MRI before performing a biopsy.

"Data suggest that serum or urine markers can avoid biopsy in at least $20 \%$ to $30 \%$ of patients, and using these markers plus multiparametric MRI can further decrease the rate of biopsy," he told listeners. "The net result is fewer negative biopsies and fewer low-risk cancers detected."

\begin{tabular}{|c|c|c|}
\hline Test & Source & Components \\
\hline PHI & Serum & PSA, fPSA, -2proPSA \\
\hline 4Kscore & Serum & $\begin{array}{l}\text { PSA, fPSA, intact PSA, kallikrein- } \\
\text { related pepitidase } 2\end{array}$ \\
\hline $\begin{array}{l}\text { ExoDx Prostate } \\
\text { (IntelliScore) }\end{array}$ & Urine & $\begin{array}{l}\text { ETS transcription factor, ERG, } \\
\text { PCA3 }\end{array}$ \\
\hline $\begin{array}{l}\text { Michigan Prostate } \\
\text { Score }\end{array}$ & Urine & PCA3, PSA, TMPSS2:ERG \\
\hline SelectMDx & Urine & mRNA DLX1, HOXC6 \\
\hline ConfirmMDx & Tissue & $\begin{array}{l}\text { DNA methylation, GSTP1, APC, } \\
\text { RASSF1 }\end{array}$ \\
\hline
\end{tabular}

Figure 1. New tests of specificity for prostate cancer screening. Abbreviations: fPDA, percent-free prostate-specific antigen; PSA, prostate-specific antigen.

Whether to use biomarkers first and then go on to MRI is still not clear. No comparative head-tohead trials are available, he noted.

\section{Active Surveillance}

Active surveillance is about timing of treatment, not foregoing treatment. The 2018 NCCN Guidelines state that active surveillance should be recommended for patients with very low risk and low risk who have a physiologic life expectancy less than 20 and 10 years, respectively. Active surveillance typically involves PSA testing every 6 months and digital rectal examination every 12 months. Follow-up prostate biopsies, use of multiparametric MRI, and genomic profiling can be used to monitor disease and the need to convert to treatment.

"In my opinion, very few men with low-risk disease should be treated," Dr. Carroll stated. "At our center, if we see a minor change in risk [during active surveillance], we don't necessarily treat that patient." He emphasized the word "active" in active surveillance, noting that PSA density-a simple, inexpensive test-is a better predictor of disease progression on active surveillance than PSA level.

Patients at high risk can be identified by adding 1 of 4 commercial genomic tests (ie, Oncotype Dx Genomic Prostate Score [GPS], Prolaris, Decipher genomic classifier, and ProMark) to the UCSF Cancer of the Prostate Risk Assessment Score (CAPRA). "One of the major anxieties is the low-risk patient who harbors high-risk disease. You can refine risk prediction when you add genomic tests to CAPRA [or other nomograms that assess disease risk]," Dr. Carroll stated.

Active surveillance has some controversial aspects. Who should have it? Is it safe for African American 
men? Are younger men appropriate candidates? In patients with a Gleason grade 3 or 4, active surveillance is not advised for those with a high PSA density, highrisk features on multiparametric MRI, adverse genomics, cribriform histology, or larger (volume) number of biopsy cores positive at diagnosis.

\section{New Approaches to the Management of Prostate Cancer}

James L. Mohler, MD, Associate Director and Senior Vice President for Translational Research, Department of Urology, and Professor of Oncology, Roswell Park Comprehensive Cancer Center, and Chair of the NCCN Prostate Cancer Panel, highlighted several important changes in the 2018 NCCN Guidelines for Prostate Cancer.

First, he criticized the adoption of unneeded technology and new drugs that come with a substantial cost burden for a small improvement in survival. "Newer treatments should be evaluated critically and compared carefully with older treatments," he said. "According to estimates compiled by Roswell Park in March 2018, the costs of new agents for castration-resistant prostate cancer are estimated to be $\$ 1.3$ million to live 2 years longer," he added (Figure 2).

Risk stratification and proper staging workup are emphasized more strongly in the 2018 version of the guidelines than in the past. "Risk stratification used to be one page," Dr. Mohler said, "and now there is an entire page for each risk group, with accompanying tables."

Further, molecular and germline testing are recommended early in the disease course for patients with a

\begin{tabular}{|l|c|c|c|}
\hline \multicolumn{1}{|c|}{ Treatment } & $\begin{array}{c}\text { FDA } \\
\text { Approval }\end{array}$ & \multicolumn{1}{c|}{ Cost } & $\begin{array}{c}\text { Added } \\
\text { Survival (mo) }\end{array}$ \\
\hline 10 cycles docetaxel & $5 / 19 / 04$ & $\$ 5,039^{\mathrm{a}}$ & 2.4 \\
\hline 1 course sipuleucel-T & $4 / 26 / 10$ & $\$ 119,300^{\mathrm{a}}$ & 4.1 \\
\hline 6 cycles cabazitaxel & $6 / 17 / 10$ & $\$ 458,581^{\mathrm{a}}$ & 2.4 \\
\hline 8 mo enzalutamide & $8 / 31 / 12$ & $\$ 88,985^{\mathrm{b}}$ & 4.8 \\
\hline 8 mo abiraterone & $12 / 10 / 12$ & $\$ 83,493^{\mathrm{b}}$ & 4.0 \\
\hline 1 course radium-223 & $5 / 15 / 13$ & $\$ 79,550^{\mathrm{c}}$ & 3.6 \\
\hline 41 mo apalutamide & $2 / 14 / 18$ & $\$ 456,674^{\mathrm{b}}$ & $\mathrm{ND}$ \\
\hline Total & & $\mathbf{\$ 1 , 2 9 1 , 6 2 2}$ & $\mathbf{2 3 . 3 +}$ \\
\hline
\end{tabular}

Figure 2. Costs of new agents for castration-resistant prostate cancer. Assumptions: Drug stopped at mean time to progression; patient completes all planned courses of all agents; patient realizes mean extension of survival reported in phase III trials; costs to patient for drug and administration from ${ }^{\mathrm{A}} \mathrm{ASP}$, $\mathrm{b} 85 \%$ AWP, or crecent actual Medicare payments (compiled by Roswell Park March 2018).

Abbreviations: ASP, average sales price; AWP, average wholesale price; $\mathrm{ND}$, not determined. strong family history, with the aim of developing more personalized recommendations. The updated guidelines define for the first time what a strong family history represents: brother, father, or multiple family members diagnosed with prostate cancer before age 60 years; known germline DNA repair abnormalities, especially BRCA2 mutations or Lynch syndrome; and $>1$ relative with breast or pancreatic cancer (suggesting BRCA2 mutation) or colorectal, endometrial, gastric, ovarian, pancreatic, small bowel, urothelial, kidney, or bile duct cancer (suggesting Lynch syndrome).

Treatment discussions should incorporate life expectancy, "which we have gotten better at estimating," he said. Life expectancy can be adjusted for individual patients by adding or subtracting $50 \%$ based on whether a patient is in the healthiest or unhealthiest quartile, respectively. For example, a 65-year-old African American man has a life expectancy of 16 years; if he is in the upper quartile of health, his life expectancy is 24 years, and if he is in the lower quartile, it is 8 years.

\section{Tumor-Based Molecular Assays for Clinically Localized Prostate Cancer}

Four tumor-based molecular assays are currently included in the NCCN Guidelines for low- or favorable intermediate-risk prostate cancer: Decipher, Oncotype DX GPS, Polaris, and ProMark. Studies have shown that these tests provide prognostic information independent of NCCN risk groups, Dr. Mohler said.

Germline testing is advised for more advanced disease, he said. Consider testing for germline and/ or somatic mutations in all men with high-risk, very high-risk, regional, or metastatic prostate cancer. Men with BRCA1/2, ATM, PALB2, or FANCA should be referred for genetic counseling and early use of platinum chemotherapy, or consider enrollment on a clinical trial. ${ }^{2}$ Men with microsatellite instability (MSI) and mismatch repair deficiency (dMMR) should be referred for genetic counseling. These patients are eligible for pembrolizumab if their disease fails to respond to androgen deprivation therapy (ADT).

The guidelines recommend against using F-18 fluorodeoxyglucose (FDG)-PET/CT to stage prostate cancer, but these more specialized PET imaging agents are becoming useful for evaluating biochemical recurrence or the possibility of metastatic disease. "The performance of these tests is poor in those who need it most; that is, men with low PSA in whom the pretest possibility of disease is low (PSA <2.0) but salvage treatment is most likely beneficial. Histo- 
Prostate Cancer and Prostate Cancer Early Detection

logic confirmation is needed wherever feasible, due to high rates of false positivity. There is a high probability of variation among equipment, protocols, interpretation, and institutions," Dr. Mohler said.

He pointed out that PET results may lead to treatment changes but not improvement in oncologic outcome, which he called the "Will Rogers effect." "Imaging is a rapidly evolving area we should watch closely. We are way behind in the United States. Europe, India, and Israel often use PET/CT or MRI for staging instead of bone scans and CT/MRI," he said.

\section{Better ADT}

Dr. Mohler also advocated for improved ADT. In castration-naïve patients, he said that luteinizing hormonereleasing hormone (LHRH) antagonists and agonists are similar. He also believes that intermittent ADT is better than continuous ADT. A meta-analysis of 6 randomized trials comparing the strategies found similar overall survival but greater toxicity with continuous ADT, and improved quality of life with intermittent ADT. "If you do it properly, there is a significant quality-of-life benefit," he noted. "We can be more intelligent and personalize ADT. If you use PSA response, we can select patients who can be off of ADT. After 18 months of ADT, patients with PSA levels $<0.2$ will do well for prolonged periods off ADT," Dr. Mohler said.

According to the 2018 NCCN Guidelines, patients with asymptomatic, castration-naïve, metastatic prostate cancer should receive intermittent ADT, making patients aware of a possible small trade-off in overall survival for improved quality of life during the off cycle. For symptomatic men, continuous ADT should be considered, but if PSA falls below $<4$, and certainly $<0.2$, intermittent ADT is reasonable. Younger, healthier men should consider docetaxel or abiraterone added to ADT, whereas older and/or unhealthier men should receive ADT.

New data from the SPARTAN trial showed that men with castration-resistant prostate cancer with no evidence of metastasis who were treated with apalutamide had a 2-year delay in developing bone metastasis versus placebo. ${ }^{4}$ Apalutamide was associated with a
$12 \%$ rate of pathologic fracture versus $6 \%$ for placebo. Apalutamide is now one of the recommended secondgeneration antiandrogens incorporated in the NCCN Guidelines. "The [guideline] panel felt the benefit of apalutamide was very important even though patients treated with it had more pathologic fractures. This needs to be further investigated. Also, longer follow-up is required to test for a survival benefit. We don't know if the delay in time-to-metastasis will translate to improved survival,” Dr. Mohler told listeners.

"Apalutamide costs approximately $\$ 11,000$ per month, so it will cost about half a million dollars per patient, since patients in the registration trial were on the drug for an average of 41 months. We need to think about financial distress," he stated.

Another new feature of the NCCN Guidelines is a distinction between management of patients with visceral metastasis versus skeletal metastasis for platinum-based versus docetaxel therapy. Patients with no visceral metastasis previously treated with abiraterone or enzalutamide can be treated with docetaxel (category 1), radium-223 for symptomatic bone metastasis (category 1), or pembrolizumab for MSI or dMMR (category 2B). Abiraterone/prednisone, enzalutamide, and sipuleucel-T are options if not previously received. Additional options are clinical trial, other secondary hormonal therapy, or best supportive care.

If patients with no visceral metastases had prior docetaxel, then abiraterone/prednisone, cabazitaxel, enzalutamide, or radium-223 for symptomatic bone metastases are all category 1 recommendations. Pembrolizumab for MSI or dMMR is a category 2B recommendation. Sipuleucel-T can be used if not previously received. Other options are clinical trial, docetaxel rechallenge, mitoxantrone/prednisone, other secondary hormone therapy, or best supportive care.

For visceral metastases, biopsy should be considered. For adenocarcinoma, docetaxel and enzalutamide are category 1 recommendations, and abiraterone/prednisone is an option. Other options are clinical trial, mitoxantrone with prednisone, and other secondary hormone therapy.

\section{References}

1. Vickers AJ, Edwards K, Cooperberg MR, Mushlin AI. A simple schema for informed decision making about prostate cancer screening. Ann Intern Med 2014;161:441-442.

2. Pritchard CC, Mateo J, Walsh MF, et al. Inherited DNA-repair gene mutations in men with metastatic prostate cancer. N Engl J Med 2016;375:443-453.
3. Dong Z, Wang $\mathrm{H}, \mathrm{Xu} \mathrm{M}$, et al. Intermittent hormone therapy versus continuous hormone therapy for locally advanced prostate cancer: a meta-analysis. Aging Male 2015;18:233-237.

4. Smith MR, Saad F, Chowdhury S, et al. Apalutamide treatment and metastasisfree survival in prostate cancer. N Engl J Med 2018;378:1408-1418. 